\title{
PRESSURE/MASS METHOD TO MEASURE OPEN POROSITY OF POROUS SOLIDS
}

\author{
Yacoubou SALISSOU and Raymond PANNETON \\ GAUS, Department of mechanical engineering, Université de Sherbrooke, Québec, Canada, J1K 2R1 \\ Yacoubou.Salissou@U.USherbrooke.ca. Ravmond.Panneton $a$ USherbrooke.ca
}

\section{INTRODUCTION}

The open porosity of a porous solid is a key parameter in the physical and acoustical modeling of porous media, especially to relate the effective properties of the fluid saturating the interconnected pores to the effective properties of the porous solid. ${ }^{1,2}$ In the past, several acoustical indirect methods have been proposed to measure this parameter ${ }^{3,4}$ However, direct methods are generally preferred. Examples of direct measurement methods include the pioneer work of Beranek, ${ }^{5}$ and its improved version standardized as ASTM D 2856-94. The method relies on the equation of state for ideal gases at constant temperature. However it is time consuming and requires several calibration of the device. Other direct measurement methods include the improvement of Beranek method proposed by Leclaire et al. ${ }^{6}$ and by Champoux et al. ${ }^{7}$ and the missing mass method proposed by Panneton and Gros ${ }^{8}$.

In this paper, a method is presented to measure the open porosity of porous solids. The method needs only a simple apparatus and its accuracy is predictable from the knowledge of the total bulk volume of the tested porous sample.

\section{THEORY}

The open porosity of porous solid is expressed as

$$
\phi=1-V_{s} / V_{t},
$$

where $V_{s}$ is the volume of the solid phase, and $V_{t}$ the total bulk volume of the porous aggregate. In general, $V_{S}$ is unknown and needs to be determined. To determine its value, the four tests presented in fig. 1 are used together with the perfect gas law relation. If the process between and during tests is very slow and room conditions are constant, isothermal condition can be assumed. The volume of the solid phase $V_{s}$ can then be expressed as [see ref $^{9}$ ]

$$
V_{s}=\left(\left(M_{2}-M_{1}\right) /\left(P_{2}-P_{1}\right)-\left(\left(M_{4}-M_{3}\right) /\left(P_{4}-P_{3}\right)\right)\right) R T
$$

where $R$ is the specific gas constant, $T$ is the temperature in Kelvin and $M_{i}$ the different masses measured on the balance.

\section{ERROR ANALYSIS}

\subsection{Applicability of the method}

As shown, to apply the proposed method, volume $V_{\mathrm{s}}$ needs first to be evaluated. Usually, the target pressures to use are low pressure $\mathrm{P}_{\text {lo }}$ and high pressure $\mathrm{P}_{\text {hi }}$ so that $P_{1} \cong P_{3} \cong P_{\text {lo }}$, and $P_{2} \cong P_{4} \cong P_{h i}$. Also, it can be easily shown that the difference $M_{i}-M_{i-1}=m_{i}-\left.m_{i-1}\right|_{i=2,4}$. Furthermore, $m_{2}-m_{4}$ yields the mass of gas occupied by the solid phase of the porous sample at high pressure (i.e., $\rho_{h i} V_{S}$, where $\rho_{h i}$ is the mass density of the gas at high pressure), and $m_{1}-m_{3}$ yields the mass of gas occupied by the solid phase at low pressure (i.e., $\rho_{l o} V_{s}$, where $\rho_{l o}$ is the mass density of the gas at low pressure). Consequently, $M_{2}-M_{4}-M_{1}+M_{3}=\left(\rho_{h i}-\rho_{l o}\right) V_{s}$ and $\rho_{l o}$ corrects for the fact that the low pressure condition is not the perfect vacuum condition. Not accounting for $m_{1}-m_{3}$ would only add a bias error proportional to $P_{l o}$ in the evaluation of $V_{s}$ and $\phi$; however it does not prevent the applicability of the method.

From the previous analysis, the most severe condition that remains for applying the method is the readability of the high pressure mass difference (i.e., $m_{2}-m_{4} \geq \varepsilon$, where $\varepsilon$ is the balance readability). This condition gives the maximum porosity the method can determine for a given set of operation conditions

$$
\phi_{\max }=1-R T_{\varepsilon} / P_{h i} V_{t} .
$$

Figure 2 gives the minimum bulk volume per balance readability as a function of open porosity for different operating conditions. It shows that larger bulk volume, larger high pressure, and gas with larger density are desirable to increase the applicability of the method, and to detect open porosity closer to unity (typical for sound absorbing porous materials).

\subsection{Precision of the method}

Using the total differential method and assuming that the errors are random and follow a normal distribution, the expected error committed on the open porosity is

$$
\delta \phi= \pm(1-\phi) \sqrt{(\delta T / T)^{2}+\left(\delta V_{t} / V_{t}\right)^{2}+(\delta Z / Z)^{2}},
$$

For a cylindrical test sample, the error on its bulk volume is given by $\delta V_{t}= \pm V_{t} \delta_{L} \sqrt{(2 / D)^{2}+(1 / H)^{2}}$, where $D$ and $H$ are its diameter and height, and $\delta_{L}$ is the uncertainty on the dimension measurement. $\delta Z$ is the error related to variable $Z$ defined, following Eq.(2), as $Z=V_{s} / R T$. Considering that the uncertainty relative to the masses and pressures are 
given, respectively, by the balance and the manometer readability $\varepsilon$ and $p$, and Assuming $P_{1}$ and $P_{3}$ are approximately equivalent and equal to low pressure $P_{l o}, P_{2}$ and $P_{4}$ are approximately equivalent and equal to high pressure $P_{h i}$, and test chamber volume $V>>V_{s}$, one obtains

$$
\delta Z \cong 2 \sqrt{\varepsilon^{2}+(p V / R T)^{2}} /\left(P_{h i}-P_{l o}\right) .
$$

The error predicted by the Eq. (4) is valid only if $V_{t} \leq V$ Figure 3 shows the expected absolute error on open porosity as a function of bulk volume per test chamber volume for three different open porosity values $(0 \%, 90 \%$, and $99 \%)$ The error generated by the individual uncertainties on the mass and pressure, the bulk volume, and the temperature are also plotted. It is noted that the precision of measurement is better when using larger bulk volume. It is also noted that for the three cases, the error is mostly controlled by the uncertainty on the mass and pressure reading. Since the error is mostly controlled by the uncertainty on the mass and pressure reading, the error on the open porosity can be estimated by the approximated expression given by

$$
\delta \phi \cong 2 \sqrt{\left((R T \varepsilon)^{2}+\left(V_{p}\right)^{2}\right)} / V_{t}\left(P_{h i}-P_{l o}\right) .
$$

One can observe that it fits well with the global error in the valid range of bulk volume $\left(V_{t} / V \leq 1\right)$

\section{EXPERIMENTAL TESTS AND RESULTS}

To validate the gas porosimeter and its precision, two tests have been performed. The first test consists in applying the method to measure the porosity of high porosity samples $(95 \%)$ of known solid phase volume $V_{s}$. The second test consists to applying the method to measure the porosity of low porosity samples (45\%) of known solid phase volume $V_{s}$. Details on test setup and procedure can be found in ref 9 . Figure 4a compares the measured standard deviation (i.e., measured error) for each of the ten samples to the theoretical prediction. It is observed that the measured error fits well with the one predicted by Eq.(6). Figure $4 \mathrm{~b}$ shows the measured mean porosity for the 30 individual tests as a function of the bulk volume to test chamber volume ratio to better visualize the scattering of the measurements around the theoretical value. One can clearly observed that the precision on the measurements improves with the bulk volume to test chamber volume ratio.

\section{ACKNOWLEDGEMENTS}

N.S.E.R.C., REGAL, and ALCAN supported this work.

\section{REFERENCES}

${ }^{1}$ Biot, J. Acoust. Soc. Am., 28, 168 (1956)

${ }^{2}$ Allard, Propagation of sound in porous media, Elsevier, NY (1993).

${ }^{3}$ Umnova et al., Appl. Acous., 66, 607 (2005).

${ }^{4}$ Fellah et al., J. Appl. Phys., 93, 296 (2003).

${ }^{5}$ L.L. Beranek, J. Acoust. Soc. Am., 13, 248 (1942).

${ }^{6}$ Leclaire et al., Rev. Sci. Inst., 74, 1366 (2003).

${ }^{7}$ Champoux et al., J. Acoust. Soc. Am., 89, 910 (1990).

${ }^{8}$ R. Panneton and E. Gros, Acta Acustica, 91, 342 (2005).

${ }^{9}$ Salissou and Panneton, J. Appl. Phys. 101, 124913 (2007)
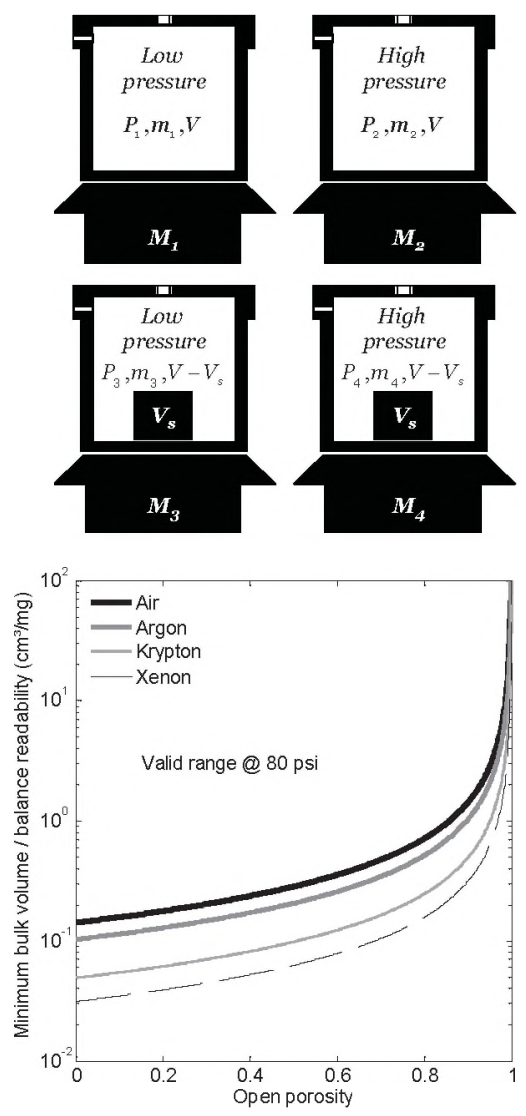

Figure 2:

Minimum bulk

volume per

balance

readability as a

function of open

porosity

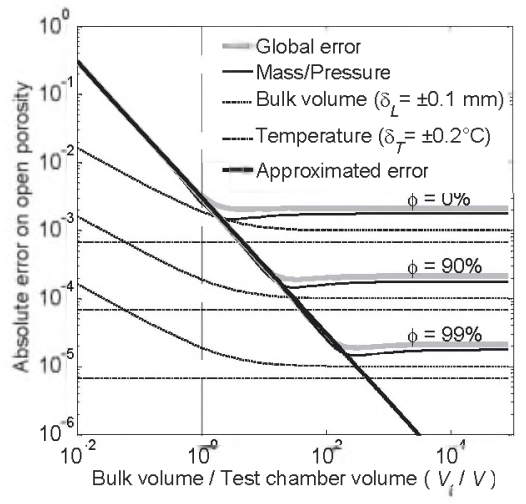

Figure 3:

Theoretical absolute error on open porosity as a function of bulk volume per test chamber volume.

Figure 4 : Experimental tests results
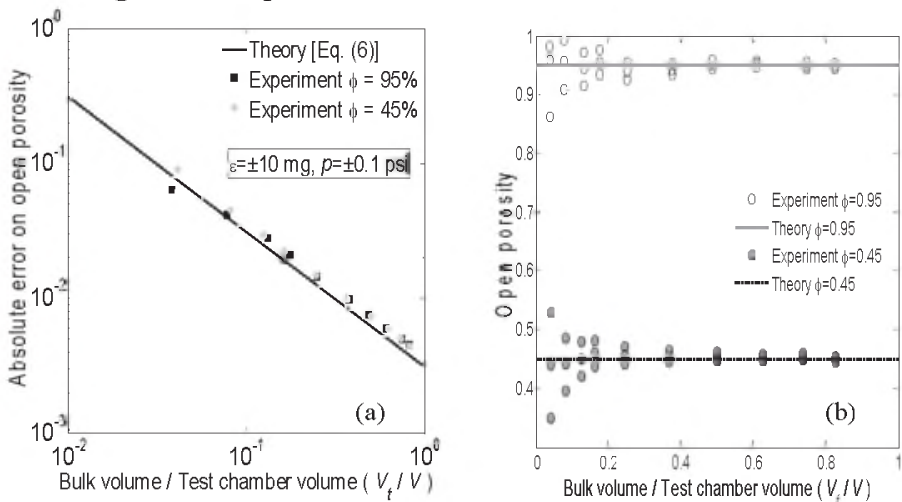